\title{
Adaptation-like effects in body weight attractiveness are not simply norm based
}

\author{
Boothroyd, L.G. ${ }^{1 *}$, Pollet, T.V. ${ }^{2}$, Qu, Y., Evans, E.H., ${ }^{3}$ Tovee, M.J. ${ }^{4}$
}

1. Department of Psychology, Durham University, South Road, Durham, DH1 3LE, UK

2. Department of Psychology, University of Northumbria, Northumberland Building, Northumberland Road, Newcastle upon Tyne, NE1 8ST.

3. School of Psychology, Newcastle University, Ridley Building 1, Queen Victoria Road, Newcastle upon Tyne, NE1 7RU

4. School of Psychology, Lincoln University, University of Lincoln, Brayford Pool, Lincoln, LN6 7TS, UK

*Author for correspondence: I.g.boothroyd@dur.ac.uk

Word count, main body: 6400

Perceptions of body weight attractiveness can be manipulated through visual exposure to bodies at one weight extreme. This implies that body weight is coded relative to a central 'norm' or prototype. Cross-cultural research, however, shows differences between groups' perceptions of body weight attractiveness in terms of preferences for specific weight ranges as well as overall differences in the perceived 'ideal' weight. Here we test for the first time whether visual exposure to body weight extremes can induce such localised changes in preferences within individuals. British and Chinese participants in Studies 1 and 3 rated 23 bodies of known BMI for attractiveness, before and after viewing underweight or overweight/obese bodies. Results of Study 1 and a combined analysis of Studies 1 and 3 together showed no evidence of significant changes in participants' 'ideal' weight, but the groups which viewed obese bodies during adaptation rated bodies of $\mathrm{BMI} 30 \mathrm{~kg} / \mathrm{m}^{2}$ significantly more positively at post-test than those who had viewed underweight bodies. In Study 2, participants viewed 48 bodies of known BMI in a randomised order. Proportion of obese bodies viewed in the preceding 10 trials predicted more positive ratings of overweight/obese bodies, but not any other weight category. Together, these data strongly suggest that we can induce 'local' changes in body ideals through exposure but do not support a strict norm-based approach.

Keywords: body weight; body preferences; adaptation; after-effects

Perceptions of body size attractiveness vary between groups and have been demonstrated to be flexible within individuals. For instance, across 26 countries, Swami et al. (2010) found sample-level ideal BMIs ranging from slim to slightly overweight on a figure choice task, and there is evidence that exposure to Western culture may drive these group differences (Boothroyd et al., 2016; Swami et al., 2010). Research into the flexibility of body attractiveness judgements has typically been conducted in the laboratory in Western, industrialised countries, and has drawn heavily on theories of perception predominantly developed in research of face perception. Early models of facial attraction emphasised the importance of prototypicality. Given that both adults and infants favour stimuli which are easier to process, it was argued that facial stimuli closer to prototype should be considered more attractive because they are easier to process (de Haan, Johnson, Maurer, \& Perrett, 2001; Langlois et al., 1987; Ramsey-Rennels \& Langlois, 2006).

Research with adults and children has confirmed that facial prototypes are flexible, and subject to visual experience. Exposure to faces manipulated in some common dimension results in a shift in preferences towards faces also manipulated in the same manner, and these preferences may be mediated by a change in perceptions of what is 'normal' (Rhodes, Jeffery, Clifford \& Leopold, 2007). This provides support for the notion that attractiveness is in some respects represented via normbased coding, i.e. with reference to a central prototype, much like other aspects of facial processing such as identity and emotion (Nishimura, Maurer, Jeffery, Pellicano, \& Rhodes, 2008; Penton-Voak, Bate, Lewis, \& Munafo, 2012; Rhodes, Jeffery, Clifford, \& Leopold, 2007). 
Similar models have been applied to our perceptions of bodies. Exposure to larger or smaller bodies, using either a simple horizontal stretch technique (Winkler \& Rhodes, 2005) or diverse real bodies of high and low BMI (Boothroyd et al., 2012; Stephen \& Perera, 2014), is associated with preferences for thinner or larger bodies at post-test. It has therefore been proposed that visual exposure to larger bodies changes the observers' reference point (or prototype) from which attractiveness judgements are derived. Cross-cultural variation in preferences has likewise been explained in part by suggesting that differences in the number of low-weight bodies in individuals' visual diets (e.g., via over representation in globalised media) has altered their central prototypes and ergo their reference point for judgements of attractiveness.

It is important to note however, that most studies on adaptation-like effects in attractiveness perceptions concentrate on a single estimate of the ideal - for instance, participants may be asked to pick a single 'most attractive' face or body from an array presented either all together or in a 'morphing' fashion (e.g., Anzures, Mondloch, \& Lackner, 2009; Rhodes et al., 2007; Stephen \& Perera, 2014; Winkler \& Rhodes, 2005), or to pick between pairs of images differing along the continuum previously manipulated from which a single overall preference score is calculated (e.g., Bestelmeyer et al., 2008; Boothroyd, Tovee, \& Pollet, 2012). Similarly perceptions of other traits associated with bodies also tend to use estimates of the boundary cut-off between different categories: Robinson \& Kirkham (2014), considered whether an image situated close to the healthy/overweight perceptual boundary was misclassified depending on exposure, while (Palumbo, Laeng, \& Tommasi, 2013) examined the categorical boundaries in perceptions of emotion/gender of faces/bodies.

Perceptions of body weight attractiveness however, do not simply consist of a single ideal. When shown bodies ranging in BMI from emaciated to morbidly obese, participants typically show an asymmetric pattern of preferences declining either side of their 'ideal'. This pattern is generally well described by a cubic function in industrialised populations (Tovee \& Cornelissen, 2001; Tovee, Maisey, Emery, \& Cornelissen, 1999). Although non-linear traits in nature are often described in terms of parabolas (e.g., stress and performance), a cubic function allows for attractiveness scores to drop more sharply on one side of ideal versus the other. This is particularly important as industrialised nations tend to favour an 'ideal' female BMI which is very close to underweight, and attractiveness ratings rapidly decline once bodies become visibly emaciated. Furthermore, cross-cultural data shows that the overall shape of this function, as well as the location of the 'peak' can be variable between groups. For instance, while there are modest differences in peak BMI preference between Zulu migrants to the UK and Britons of African descent, there are more marked differences in the preference function shape, such that Zulu migrants are considerably more positive about overweight/obese bodies than British participants, giving obese bodies significantly higher ratings, while they are similarly averse to very underweight figures (Tovee, Swami, Furnham, \& Mangalparsad, 2006). Similar patterns have been observed comparing rural Nicaraguans with, versus without, access to globalised media (Boothroyd et al., 2016) and the authors of both studies hypothesised that the differences they observed between groups were driven by individual-level cultural experience. No one has yet tested, however, whether such differences in preferences across the weight spectrum, as well as in central ideals, can be induced within a single experimental setting. Such evidence is crucial if we are to accept the hypothesis that differences in preference function shape between groups is driven by individual level visual experience.

The current studies therefore sought to calculate preference functions in the context of an adaptation paradigm. We have previously published data showing that viewing 40 overweight/obese or underweight figures leads to, respectively, a weakening or a strengthening of preferences for thinner bodies in an extended forced choice preference task (Boothroyd et al., 2012). This effect was found regardless of whether the bodies were positively valenced (which might also induce associative learning) or relatively negatively valenced, suggesting that the effect is likely driven in part by changes in prototype via adaptation-like effects. In Study 1 of the current paper, we repeated the positively valenced conditions from this previous experiment, but at pre- and post-test participants rated 23 bodies of known BMI, and we calculated their preference function rather than a simple preference score. If perceptions of body attractiveness are anchored to a norm, we would predict that exposure 
to bodies of high or low weight should shift the entire preference function in the direction of the new updated prototype, without affecting the shape of that function.

To illustrate this with hypothetical data, Figure 1, panel a, shows the cubic function arising from a hypothetical pattern of data for bodies with BMIs from 11 to 40 that peaks at a BMI of 20 (solid blue line), with a 2 BMI-point shift upwards (dashed red line, peaks now at 22) but no other change, except for the lowest two BMI points remaining 'at floor'. Alternatively, if body weight attractiveness perceptions can be affected 'locally' within the spectrum, exposure to high BMI bodies might result only in a change in preferences for larger bodies. Figure 1 panel $b$ shows the same hypothetical data with an increase of 1 (out of 7) on ratings of all bodies over BMI 30.

In Study 2 we analysed trial-level data from participants viewing 48 randomised bodies of known BMI in order to determine whether adaptation effects could be observed at a trial-by-trial level with neutral stimuli. Finally, in Study 3 we combined these methods. All three studies utilised British samples, however Studies 1 and 3 also recruited Chinese samples in order to consider how our results generalised to a cultural setting with a documented difference in body ideals (Chinese samples prefer slimmer bodies than British samples: Mo et al., 2014).

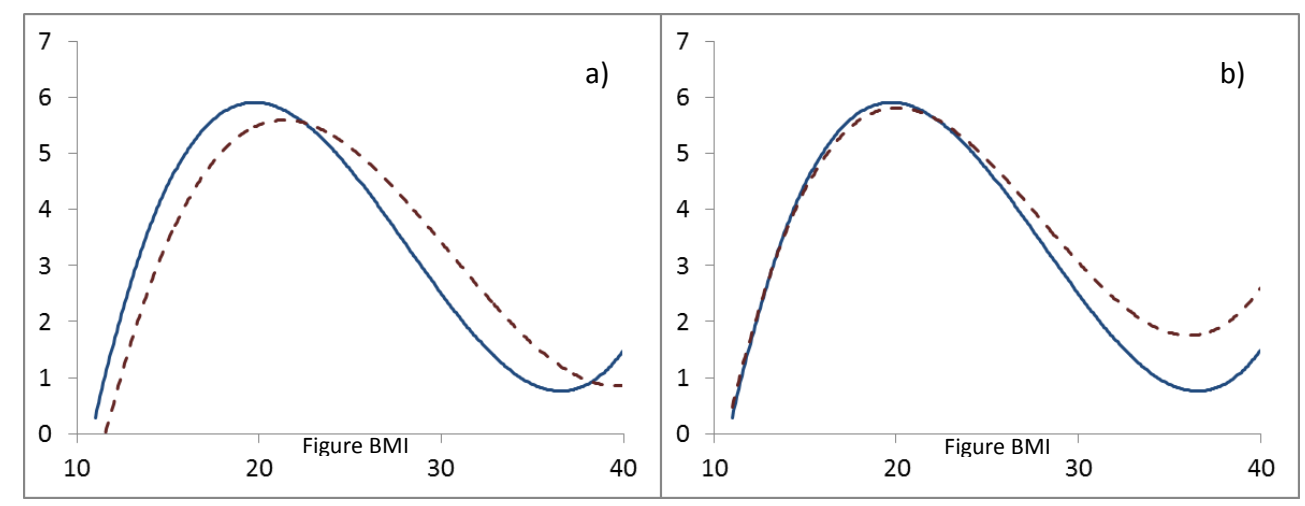

Figure 1. Hypothetical effects of a. shifting peak weight ideal 2 BMI points upwards, or $b$. increasing attractiveness of bodies with BMI 30+ by 1 Likert scale point. Note that in panel a, ratings of underweight bodies are decreased, ratings of overweight/obese bodies are increased, and there is a rightward shift in function apex. In panel $b$, there is an increase in ratings of overweight bodies but no change in ratings of underweight bodies or in function apex. (See ESM 1 for data used to produce these figures.) Note also that using preference at BMI 30 as an outcome avoids problems with increasing predicted preferences at $B M I 40$ as an artefact of the cubic function.

\section{Methods}

\section{Study 1}

Participants. We recruited three samples of participants in the UK and China.

Sample 1 participants were recruited through the Psychology undergraduate participant pool (with partial course credit) and by circular email to the Mathematics undergraduates at a northern English university. Participants used their own or university computers, were directed to the study webpage and indicated consent by button click. Participants who did not complete the full study were excluded. The final sample consisted of 26 men and 63 women aged 18-28 ( $M=19.3$ years).

Sample 2 participants were recruited through CrowdFlower or the experimenter's website, and all were resident in the UK at the time of testing. Participants were paid following completion according to the site's protocols. Participants used their own computers, were directed to the study webpage from the CrowdFlower testing site or the experimenter's website, and indicated consent by button 
click. Participants who did not complete the full study, or who reported non-British nationality, were excluded. The final sample consisted of 35 men and 40 women aged 20-62 ( $M=39.1$ years).

Sample 3 participants were recruited by word of mouth in the city of Qingdao in the Shandong province of Eastern China. Sixty participants were tested in situ by QY using a laptop computer. Following exclusions for non-viable functions (see below), the sample consisted of 19 men and 29 women aged $18-30$ ( $M=23.8$ years).

Preference task. Participants rated two sets of 23 bodies of known BMls for attractiveness on a 1-7 Likert scale from 'Very unattractive' to 'Very attractive'. Stimuli all showed adult British women photographed under standard conditions while wearing grey leotards and with faces blocked out. Stimulus BMIs ranged from 12 to 38 . All 46 stimuli were initially ranked by BMI and then assigned alternately into two sets; set 1 were rated at pre-test and set 2 were rated at post-test.

Manipulation task. After rating the first set of 23 bodies, participants viewed and rated 40 further images for attractiveness in the same manner. These images were of either underweight women ('standard' slim catalogue models and beauty queen contestants), or overweight/obese women (plus size models and plus size beauty queen contestants). This task was previously used by Boothroyd et al. (2012) as their first two experimental conditions.

Procedure. All participants reported their gender identity and age, either in an online form (Samples 1 and 2), or on the researcher's computer (Sample 3). British participants then reported their sexual orientation on a 1-7 Likert scale from exclusively heterosexual to exclusively homosexual and those scoring over 3 were excluded. It was not felt possible to ask the Chinese participants about sexual orientation in person. British participants also completed a body satisfaction measure for another study prior to participation.

Participants in Samples 1 and 2 were divided into two conditions ('underweight'/'overweight') by indicating their birthday (whether they were born on the $1^{\text {st }}-15^{\text {th }}$ or $16^{\text {th }}-31^{\text {st }}$ ) and clicking the appropriate link; group allocations were counterbalanced over recruitment. Participants in Sample 3 were allocated alternately, within gender, to the two conditions. The participants rated Set 1 preference task stimuli, followed by all manipulation phase stimuli, followed by the Set 2 preference task stimuli. Within each set, stimuli were presented in a random order and at a size of $400 \times 600$ pixels.

Data processing. Each participant's ratings for each body at pre-test were regressed onto image BMI and the cubic function was calculated in Microsoft Excel using the LINEST function. From this, the BMI value representing the apex in the function was calculated to 3 decimal points. Following Tovee et al. (2006), we also calculated the predicted attractiveness rating each participant would have given to an image with BMI of 30 . This same analysis was repeated with the post-test data. Where a participant failed to produce a viable function their data were excluded. Non-viable functions were evident where our algorithm yielded an 'error' term or a predicted peak preference outside the range of BMI in our stimuli; scatterplots for these cases were checked manually and typically showed apparently random responses by the participant. Initial analyses showed that at baseline, peak BMI preferences did not significantly correlate with age in any sample, and Preference at BMI 30 did not significantly correlate with age in the UK student and Chinese samples (all $r<.2$, all $p>.24$ ). There was a correlation trending toward significance between age and preference for BMI 30 in the UK non-student sample $\left(r_{76}=.19, p\right.$ $=.096$ ) and so age was included as a covariate in analyses for this sample.

\section{Results and discussion}

Mean preferences for each image at pre- and post-test are shown in Figure 2. To assess changes in preference function shape and location, data were analysed in random intercept models, using the ImerTest package (Kuznetsova, Brockhoff, \& Christensen, 2016) in R version 3.3.3 (R Development Core Team), with preferences (as calculated above) clustered into participants. Time (pre- vs posttest) was entered as a lower-level predictor, while condition (overweight/obese vs underweight 
models) and gender (male vs female) were entered as participant level predictors. The interactions between all three predictors were modelled. For sample 2, age was also added as a participant-level covariate. Results are summarised in Table 1; code and full analyses are provided in the Supplementary material.

Under simple norm-based coding of attractiveness (i.e. that the 'ideal' body is represented relative to a single 'normal' prototype), we would expect adaptation to result in a horizontal shift of the entire preference function towards either larger or smaller bodies depending on the weight status of the adapting stimuli. As such, peak BMI preferences should show significant change across groups. For peak BMI preferences, there were no such interactions between time and condition for any of the three samples (see Table 1), except for a higher order interaction between time, condition and sex for Sample 2. When the data were split by participant gender and the analyses re-run, the two-way interaction between time and condition emerged as significant in the male participants ( $B=.938, \mathrm{SE}=$ $.315, p=.005$ - those who saw larger bodies had a higher peak preference at post-test) but not female $(\mathrm{B}=-.400, \mathrm{SE}=.500, p=.428)$.
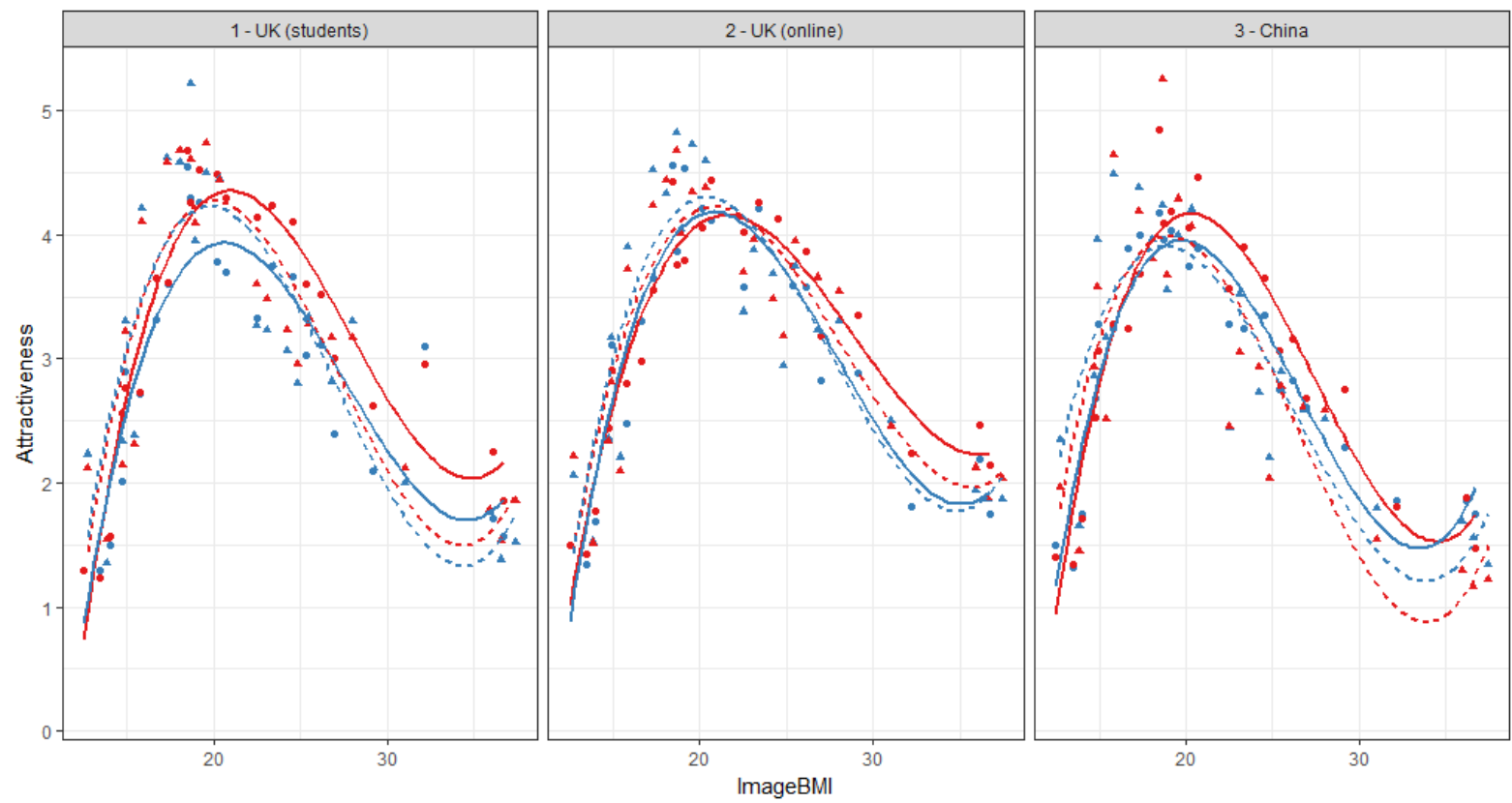

Figure 2. Mean ratings for each body plotted against image BMI for those in the overweight/obese bodies condition (red) and the underweight bodies condition (blue) at pre-test (dashed lines and triangles) and post-test (solid lines and circles) for each Study 1 sample.

We found more consistent evidence for a change in the shape of the preference functions. Both samples 1 and 3 produced a significant interaction between time and condition for Preference at BMI 30 (Sample 1: $\mathrm{B}=.494$, $\mathrm{SE}=.175, p=.006$; Sample 3: $\mathrm{B}=.772$, $\mathrm{SE}=.351, p=.032$ ). As can be seen in Figure 2, although participants in the two groups tended not to show differences in ratings in the lower half of the distribution at post-test, those who viewed overweight/obese bodies during the manipulation phase rated larger bodies as more attractive at post-test relative to pre-test, compared to the change amongst those who had been shown underweight bodies in the manipulation phase. (Note that because different images were used at pre- and post-test we do not necessarily expect the functions to entirely overlap even if there is no underlying change in internal representations; results are therefore best understood in terms of change over time relative to the other group.)

Although there was no 2-way interaction in Sample $2(B=-.227, S E=.187, p=.230)$, there was a significant three-way interaction with gender $(\mathrm{B}=.633, \mathrm{SE}=.274, p=.024)$ and again, the two-way interaction between time and condition was present in the male participants $(B=.406, S E=.145$, $p=.008$ ) but not the female participants ( $\mathrm{B}=-.227, \mathrm{SE}=.223, p=.315)$. As in Samples 1 and 2 the effect 
was largely driven by change in the overweight/obese bodies condition rather than the underweight bodies condition.

Further models tested whether there was any change in ratings of underweight bodies (predicted preference at BMI 16) to assess whether there was a local impact of viewing slim bodies. Although in Sample 1 there was a significant interaction between time and group for this variable $(B=-.393, S E=$ $.149, p=.010)$, it was superseded by a higher order interaction with gender $(B=-.543, S E=.273$, $\mathrm{p}=.050$ ). On further analysis, neither gender showed a significant time by condition interaction. Although both showed negative coefficients, indicating that preferences for BMI 16 were lower in the overweight/obese bodies condition at post-test compared to the underweight bodies condition, the interaction appears to have arisen due to this effect being stronger in men $(B=-.195, S E=.125$, $\mathrm{p}=.128)$ than women $(\mathrm{B}=-.026, \mathrm{SE}=.163, \mathrm{p}=.872)$.

Table 1. Results for Study 1 linear mixed models showing effects of adaptation period on peak BMI preference and predicted preference rating for a body of BMI 30. The critical interaction which tests the hypothesised adaptation effects is highlighted in bold.

Sample 1 - UK Students

Intercept
Group
Time
Sex (male)
Group x Time
Group x Sex
Time x Sex
Group x Time x Sex

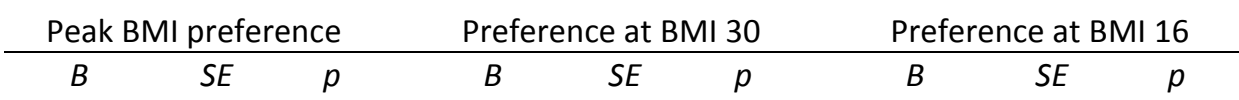

$\begin{array}{ccc}19.758 & 0.342 & .000 \\ 0.252 & 0.453 & .578 \\ 0.524 & 0.283 & .067 \\ 0.200 & 0.600 & .740 \\ \mathbf{0 . 0 2 4} & \mathbf{0 . 3 7 4} & .949 \\ 0.355 & 0.831 & .670 \\ -0.050 & 0.496 & .920 \\ 0.680 & 0.687 & .325\end{array}$

$\begin{array}{llllll}0.849 & 0.172 & .000 & 2.659 & 0.149 & .000 \\ 0.109 & 0.227 & .631 & 0.048 & 0.197 & .808 \\ -0.157 & 0.132 & .238 & -0.473 & 0.113 & .000 \\ -0.438 & 0.301 & .148 & -0.497 & 0.261 & .059 \\ \mathbf{0 . 4 9 4} & \mathbf{0 . 1 7 5} & .006 & \mathbf{0 . 3 9 3} & \mathbf{0 . 1 4 9} & .010 \\ 0.401 & 0.417 & .339 & 0.050 & 0.362 & .889 \\ 0.310 & 0.232 & .184 & 0.309 & 0.197 & .121 \\ -0.282 & 0.321 & .382 & -0.543 & 0.273 & .050\end{array}$

Sample 2 - UK online

Intercept
Age
Group
Time
Sex (male)
Group x Time
Group x Sex
Time x Sex
Group x Time x Sex

$\begin{array}{ccc}19.318 & 0.801 & .000 \\ 0.025 & 0.017 & .151 \\ 0.861 & 0.564 & .130 \\ 0.974 & 0.302 & .002 \\ 0.388 & 0.585 & .509 \\ -0.400 & 0.417 & .340 \\ -0.713 & 0.831 & .393 \\ -0.616 & 0.433 & .159 \\ 1.339 & 0.610 & .031\end{array}$

$\begin{array}{llllll}1.615 & 0.454 & .001 & 3.473 & 0.377 & .000 \\ 0.016 & 0.010 & .109 & 0.004 & 0.008 & .673 \\ 0.699 & 0.314 & .028 & -0.089 & 0.259 & .731 \\ 0.224 & 0.136 & .103 & -0.342 & 0.104 & .002 \\ -0.008 & 0.325 & .979 & -0.381 & 0.269 & .160 \\ -0.227 & 0.187 & .230 & -0.026 & \mathbf{0 . 1 4 3} & .854 \\ -0.573 & 0.462 & .218 & 0.046 & 0.382 & .905 \\ -0.140 & 0.195 & .475 & 0.207 & 0.149 & .169 \\ 0.633 & 0.274 & .024 & -0.169 & 0.210 & .424\end{array}$

Sample 3 - China

\begin{tabular}{lccccccccc} 
Intercept & 18.076 & 0.551 & .000 & 1.279 & 0.276 & .000 & 3.779 & 0.241 & .000 \\
Group & 1.069 & 0.779 & .173 & 0.307 & 0.391 & .435 & 0.007 & 0.341 & .983 \\
Time & 1.485 & 0.443 & .001 & 0.261 & 0.248 & .298 & 0.011 & 0.195 & .954 \\
Sex (male) & 1.988 & 0.793 & .014 & 0.748 & 0.398 & .063 & -0.387 & 0.347 & .268 \\
Group x Time & $\mathbf{0 . 3 2 3}$ & $\mathbf{0 . 6 2 6}$ & .608 & $\mathbf{0 . 7 7 2}$ & $\mathbf{0 . 3 5 1}$ & .032 & $-\mathbf{0 . 1 1 4}$ & $\mathbf{0 . 2 7 6}$ & .681 \\
Group x Sex & -2.260 & 1.103 & .044 & -1.100 & 0.553 & .050 & -0.098 & 0.483 & .841 \\
Time x Sex & -0.091 & 0.637 & .887 & 0.017 & 0.357 & .962 & -0.528 & 0.281 & .065 \\
Group x Time x Sex & -0.189 & 0.886 & .832 & -0.434 & 0.497 & .387 & 0.269 & 0.391 & .494 \\
\hline
\end{tabular}


Overall, the data show good support for an impact of viewing large bodies on preferences for bodies with a BMI of $30 \mathrm{~kg} / \mathrm{m}^{2}$, but no significant evidence of shifts in peak BMI preference or preferences at a $\mathrm{BMI}$ of $16 \mathrm{~kg} / \mathrm{m}^{2}$. These results run counter to the predictions derived from a single norm-based representation of body attractiveness and show that cultural variations previously seen in preference function shape can be experimentally induced. It is possible, however, that our results are affected by the valence of our adapting stimuli. While Boothroyd et al. (2012) found both positively valenced stimuli (models) and neutrally valenced stimuli (eating disordered patients in leotards) had equal effects on participants' thinness-preference (see also Stephen and Perera, 2014), we only used positively valenced stimuli in our manipulation phase. As such, our results may instead reflect higher level associative learning of a positive association between weight and cues to status in the overweight/obese bodies condition, leading to a localised 'lift' in attitudes to larger bodies. The lack of any clear benefit from viewing thin models on ratings of underweight bodies may be due to our participants' media environment already being saturated with glamourous slim models. This is especially pertinent given the fact that in their second study, Boothroyd et al paired positively valenced stimuli with negatively valenced stimuli of the opposite size and still found effects for participants who had seen the counter-cultural 'large is good, thin is bad' condition, but not those in the opposite condition. One aim of Study 2 therefore was to investigate adaptation effects in a manner which made such associative learning unlikely.

\section{Study 2}

The results of Study 1 not only required that we examine the impacts of body adaptation in a manner which was unlikely to induce valence learning, but also suggested that the effects of adaptation paradigms on attractiveness ratings may be 'localised' rather than generalised across the entire spectrum along which the manipulation has taken place. In Study 2, we did not simply repeat Study 1 with the non-aspirational stimuli from Boothroyd et al (2012) as these stimuli were in fact drawn from the 50 bodies utilised at pre- and post-test in Study 1 and would have required participants rating the same bodies in both the manipulation and test phases. Instead, we had all participants rate all 50 bodies in a randomised order and examined the impact of the preceding 10 bodies on the attractiveness ratings of each subsequent image. Each participant rated each body following a different combination of obese, underweight and typical weight bodies; as such we can compare instances where the image was preceded by a large number of obese bodies, or underweight bodies. If, as Study 1 suggests, the effects of our adaptation paradigm were localised, then we would predict that exposure to more overweight/obese bodies would increase relative attractiveness ratings of large bodies, but not slim bodies, and vice versa. In contrast, if stimuli were updating a single prototype from which attractiveness were coded, we would predict that exposure to a large number of overweight/obese bodies would increase ratings of larger bodies and decrease ratings of smaller bodies (and again, vice versa).

\section{Methods}

Participants. Fifty-four British-resident adults ( 23 female) were recruited through CrowdFlower. Participants were paid following completion according to the site's protocols. Participants used their own computers, were directed to the study webpage from the CrowdFlower testing site or the experimenter's website, and indicated consent by button click. Participants who did not complete the full study, or whose data did not produce a viable function were excluded. The final sample consisted of 27 men and 17 women aged 19-70 (mean 36.16 years).

Procedure. Participants reported their gender identity and age. Participants then rated 48 bodies of known BMls, used in the preference tasks in Study 1, for attractiveness on a 1-7 Likert scale from 'Very unattractive' to 'Very attractive'. Stimuli were presented in a random order to each participant.

Data processing. As above, the cubic relationship between image BMI and attractiveness score was calculated for each participant. The residuals from that function were then calculated for each image 
and these data were used in the analyses below. For each trial within each participant, the proportion of the preceding 10 images with a BMI exceeding $30 \mathrm{~kg} / \mathrm{m}^{2}$ was calculated (Proportion obese), and the proportion of the preceding 10 images with a BMI lower than 18 was likewise calculated (Proportion underweight). Data from the first 10 trials for each participant was excluded from later analyses.

Finally, each image was classified as underweight $\left(B M I<18 \mathrm{~kg} / \mathrm{m}^{2}\right)$, healthy weight $\left(\mathrm{BMI} 18-25 \mathrm{~kg} / \mathrm{m}^{2}\right)$ or overweight (BMI $\left.>25 \mathrm{~kg} / \mathrm{m}^{2}\right)$.

\section{Results and discussion}

Data were entered into a linear mixed model using the ImerTest package in R. Participant and image were entered as cross-classified first level random factors. Participant sex was entered as a fixed factor, and the interaction terms between Body category and both 'Proportion obese' and 'Proportion underweight' were also entered.

A significant interaction between Proportion obese and Body category arose (see Table 2). While there were no significant effects of Proportion obese for the underweight or healthy weight trials, there was a significant positive relationship between proportion obese and residual attractiveness of overweight/obese images $(\mathrm{B}=.62, \mathrm{SE}=.25, \mathrm{p}=.01$ ). As such, the more obese images a participant had recently seen, the higher they rated overweight/obese images relative to the predicted score for that participant viewing that image. There was no effect of viewing underweight bodies on subsequent ratings of bodies in any weight category.

Table 2. Mixed model effects of previous body BMIs on residual attractiveness of images at trial level

\begin{tabular}{lccccc} 
& $\boldsymbol{B}$ & $\boldsymbol{S E}$ & $\boldsymbol{d f}$ & $\boldsymbol{t}$ & $\boldsymbol{P}$ \\
\hline (Intercept) & -0.02 & 0.11 & 98 & -0.17 & 0.86 \\
Sex & -0.01 & 0.03 & 1618 & -0.21 & 0.83 \\
Proportion obese & & & & & \\
$\quad$ x Healthy weight body & -0.03 & 0.24 & 1461 & -0.11 & 0.91 \\
$\quad$ x Overweight/obese body & $\mathbf{0 . 6 2}$ & $\mathbf{0 . 2 5}$ & $\mathbf{1 3 3 7}$ & $\mathbf{2 . 4 6}$ & $\mathbf{0 . 0 1}$ \\
$\quad$ x Underweight body & -0.28 & 0.25 & 1471 & -1.12 & 0.27 \\
Proportion underweight & & & & & \\
$\quad$ x Healthy weight body & 0.10 & 0.20 & 1270 & 0.50 & 0.62 \\
$\quad$ x Overweight/obese body & -0.07 & 0.22 & 993 & -0.31 & 0.76 \\
$\quad$ x Underweight body & $-\mathbf{0 . 2 5}$ & $\mathbf{0 . 2 1}$ & $\mathbf{1 3 0 0}$ & $\mathbf{- 1 . 2 0}$ & $\mathbf{0 . 2 3}$ \\
$* p$ & & & & &
\end{tabular}

These results are consistent with those in Study 1 in showing that viewing obese bodies has a specific impact on perceptions of bodies with similar BMIs (i.e. overweight/obese bodies are viewed more positively) but no impact on bodies elsewhere on the BMI range. It is also consistent in failing to find an impact of viewing underweight bodies, again perhaps because our participants' media environment is so saturated with images of underweight women. These results therefore continue to point to body attractiveness representations being distinct at different weight categories rather than coded with reference to a single prototype.

It remains the case, however, that these images were shown in a randomised order and because there were only 10 obese images in the dataset to begin with, relatively few trials involved participants having seen more than 5 obese images in the last 10 trials. As such we may have been underpowered to detect effects in other interactions. 


\section{Study 3}

Study 3 sought to build on Study 2 by artificially increasing the number of underweight or obese images viewed by participants. It combined the designs of Studies 1 and 2, by asking participants to rate bodies in a seemingly continuous stream of similar stimuli (all the grey leotard images from Study 2) but a middle section consisting of predominantly obese or underweight images in a more similar density to Study 1. In order to achieve this, the images which had been rated at pre-test in Study 1 were used at both pre- and post- test in Study 3, with changes in border colour and/or horizontal flipping being used to ensure that no identical image was presented to participants (i.e. they might see image 6 in its normal appearance at pre-test, but with a border at post-test). The images previously shown at post-test in Study 1 were then utilised to create a battery of predominantly high or low weight images, again by adding purple borders and by horizontally flipping the images. For both conditions, participants saw 25 images in the target weight category in this section, with an additional 11 bodies from the $17-28 \mathrm{~kg} / \mathrm{m}^{2} \mathrm{BMI}$ range as distractors.

If the results of Study 1 hold with non-aspirational adaptation images, and if the results of Study 2 hold when a high density of obese/underweight images are seen by all participants, we would thus predict that localised preferences should show a change following the adaptation phase (i.e. that viewing a high density of obese figures should result in increased attractiveness of bodies at a BMI of $30 \mathrm{~kg} / \mathrm{m}^{2}$ ). Participants were again recruited from both the UK and China.

\section{Methods}

Participants. Both samples were recruited simultaneously through Prolific; budget was allocated to the experimenters' account for 200 participants, aiming for 100 in each sample, and recruitment ceased when all budget was spent which resulted in samples biased towards British participants. Participants were paid following completion according to the site's protocols. Participants used their own computers, were directed to the study webpage from the Prolific testing site, and indicated consent by button click.

Sample 1 participants were all resident in the UK at the time of testing. 5 participants reporting Chinese nationality, and three reporting Australian, Moroccan and Estonian nationality were excluded. Participants reporting their sexual orientation as greater than 3 on a 7 point Kinsey scale (i.e. strictly bisexual or predominantly/exclusively homosexual), and those who failed to produce viable preference functions were also excluded, leaving 117 participants (37 men) who reported being British or Irish with a mean age of 37 years $(S D=12.2)$. 109 participants reported White ethnicity.

Sample 2 participants were all resident in China at the time of testing. Participants reporting European nationality, reporting their sexual orientation as greater than 3 on a 7 point Kinsey scale, and those who failed to produce viable preference functions were excluded leaving a total of 44 participants ( 22 men) with a mean age of 27 years $(S D=7.9) .42$ participants reported Chinese ethnicity.

Stimuli and procedure. All images were duplicated with versions showing a purple border, a red border, and horizontally flipped versions, thus giving six available stimuli per original image (plain, red and purple border, in original or flipped presentation). Two versions of each image from Set A were then assigned randomly for use, in pre-test or post-test, while the remainder were not used. 11 images in Set B were randomly allocated as distractors, consisting of one image with a BMI of 17.49, and two with each of: 18.05, 20.33, 22.49, 25.49 and 28.01. The remaining 25 trials in the manipulation phase consisted of images from Set B with BMls either below 16 or over $30 \mathrm{~kg} / \mathrm{m}^{2}$. Mean BMI of images in the high weight manipulation phase was $31.06 \mathrm{~kg} / \mathrm{m}^{2}$, while for the low weight manipulation phase it was $16.78 \mathrm{~kg} / \mathrm{m}^{2}$. Participants saw all 82 trials in one apparently continuous stream and rated each image for attractiveness on a 1-7 Likert scale.

\section{Results and discussion}


Data were initially analysed following identical procedures to Study 1. Peak BMI and preference at $\mathrm{BMI} 30 \mathrm{~kg} / \mathrm{m}^{2}$ and $16 \mathrm{~kg} / \mathrm{m}^{2}$ were calculated for all participants and entered in mixed effect regressions controlling for age as both studies had a broad age sample. Although the overall functions (see Figure 3) appeared to show viewing underweight bodies increased ratings of healthy weight in the Chinese sample and depressed ratings of healthy weight bodies in the UK sample, there were no significant interactions between group and time for any of the outcome variables (see Table 3 for results). The Chinese sample, however, did show an interaction in the predicted direction which approached significance for peak BMI preference ( $B=1.494, p=.07$ - note 2-tailed $p$ value used), such that those in the obese body condition preferred a body on average 1.14 BMI points higher at postversus pre-test, while those in the underweight bodies condition showed a drop of -.36 BMI points in the model.

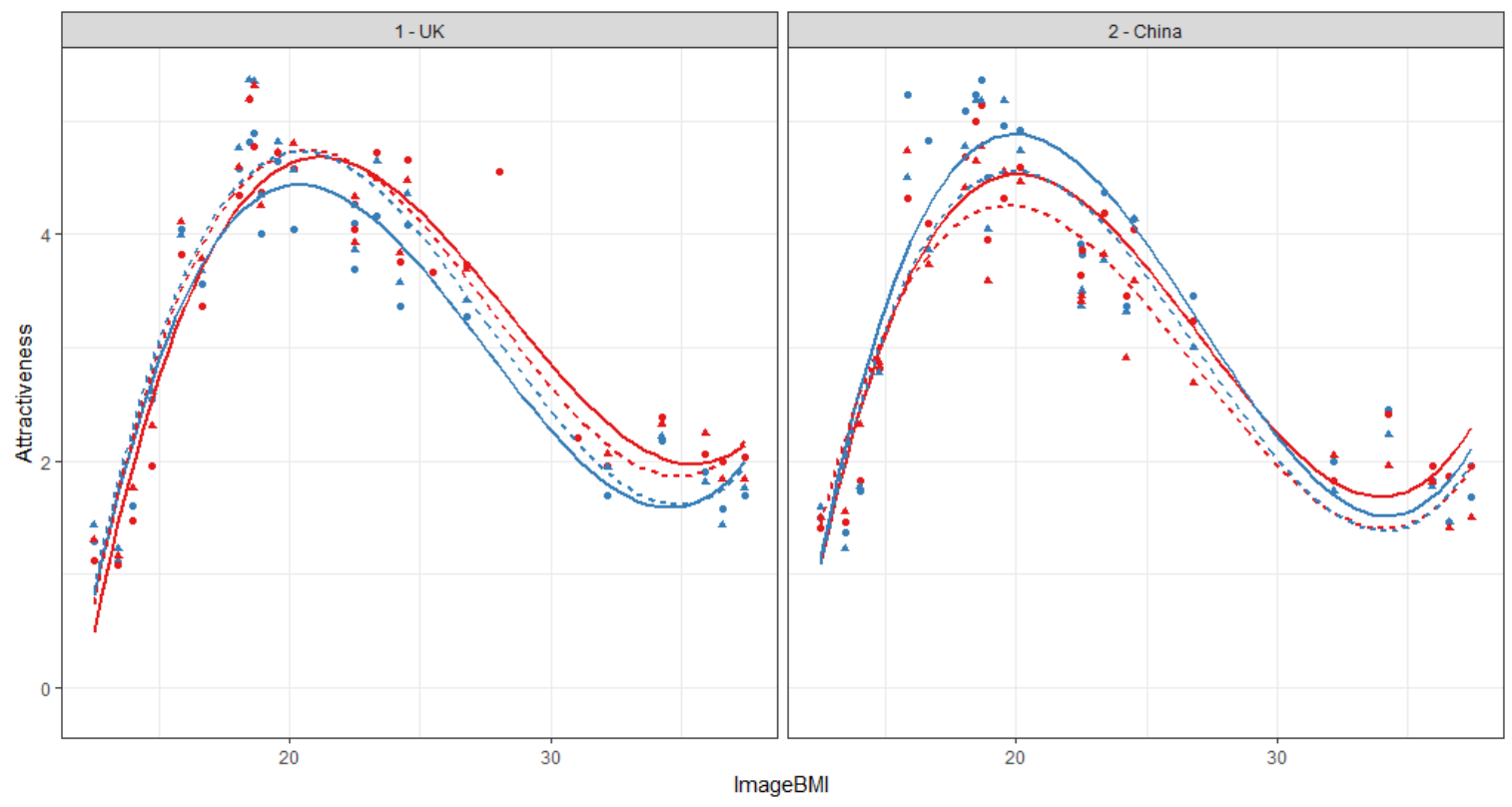

Figure 3. Mean ratings for each body plotted against image BMI for those in the obese bodies condition (red) and the underweight bodies condition (blue) at pre-test (dashed lines and triangles) and post-test (solid lines and circles) for each Study 3 sample.

These results were counter to those observed in Study 1 where an impact of large adapting stimuli on preferences for large bodies was seen in all three samples (either in the full sample or in one sex), which raises the possibility that those Study 1 results were driven by the positive valence of the adapting stimuli. Such an explanation, however, would not explain the results of Study 2 in which all stimuli were non-aspirational. An alternative possibility is that the lower number of adapting stimuli and inclusion of distractors in the current experiment weakened the strength or longevity of the adaptation effect such that it was not evident when considering the indices used in Study 1 . We therefore ran additional exploratory analyses on manipulation phase trial level data from all participants together, to determine whether increased exposure to the target body weight changed preferences over the course of the manipulation phase itself, even if that effect did not persist at posttest. 
Table 3. Results for Study 3 random intercept models showing effects of adaptation period on peak $B M I$ preference and predicted preference rating for a body of $B M I 30$ or $16 \mathrm{~kg} / \mathrm{m}^{2}$. The critical interaction which tests the hypothesised adaptation effects is highlighted in bold.

\begin{tabular}{|c|c|c|c|c|c|c|c|c|c|}
\hline \multirow{3}{*}{ Sample 1 - UK Online } & \multicolumn{3}{|c|}{ Peak BMI preference } & \multicolumn{3}{|c|}{ Preference at BMI 30} & \multicolumn{3}{|c|}{ Preference at BMI 16} \\
\hline & $B$ & $S E$ & $p$ & $B$ & $S E$ & $p$ & $B$ & $S E$ & $p$ \\
\hline & & & & & & & & & \\
\hline Intercept & 20.665 & 0.503 & .000 & 2.019 & 0.443 & .000 & 3.331 & 0.198 & .000 \\
\hline Age & 0.000 & 0.012 & .983 & 0.000 & 0.011 & .979 & 0.005 & 0.005 & .278 \\
\hline Group & 0.455 & 0.408 & .267 & 0.440 & 0.350 & .210 & -0.030 & 0.160 & .853 \\
\hline Time & -0.084 & 0.234 & .720 & -0.274 & 0.171 & .112 & -0.089 & 0.088 & .316 \\
\hline Sex (male) & -0.195 & 0.483 & .687 & -0.229 & 0.413 & .580 & -0.063 & 0.189 & .739 \\
\hline Group x Time & 0.247 & 0.385 & .522 & 0.375 & 0.283 & .187 & 0.027 & 0.146 & .851 \\
\hline Group x Sex & 0.062 & 0.712 & .931 & -0.109 & 0.610 & .859 & -0.144 & 0.278 & .606 \\
\hline Time $x$ Sex & 0.087 & 0.454 & .848 & 0.227 & 0.333 & .496 & -0.304 & 0.171 & .079 \\
\hline Group x Time x Sex & 0.637 & 0.672 & .345 & 0.224 & 0.493 & .650 & 0.048 & 0.254 & .850 \\
\hline Sample 2 - China Online & & & & & & & & & \\
\hline Intercept & 20.312 & 1.012 & .000 & 1.442 & 0.737 & .055 & 3.694 & 0.416 & .000 \\
\hline Age & 0.016 & 0.034 & .634 & 0.005 & 0.025 & .828 & -0.006 & 0.014 & .666 \\
\hline Group & -1.144 & 0.771 & .142 & -0.115 & 0.564 & .839 & 0.293 & 0.312 & .350 \\
\hline Time & -0.356 & 0.569 & .535 & -0.125 & 0.425 & .770 & 0.252 & 0.214 & .244 \\
\hline Sex (male) & -1.234 & 0.882 & .166 & -0.705 & 0.645 & .278 & -0.335 & 0.357 & .351 \\
\hline Group x Time & 1.494 & 0.791 & .065 & 0.250 & 0.591 & .674 & -0.411 & 0.297 & .172 \\
\hline Group x Sex & 1.602 & 1.220 & .193 & 0.498 & 0.892 & .578 & -0.552 & 0.493 & .266 \\
\hline Time $x$ Sex & 1.112 & 0.909 & .227 & 0.533 & 0.680 & .437 & 0.361 & 0.342 & .296 \\
\hline Group x Time x Sex & -1.029 & 1.258 & .417 & -0.189 & 0.940 & .842 & -0.137 & 0.472 & .773 \\
\hline
\end{tabular}

Studies 1 and 3

$\begin{array}{llllllllll}\text { Intercept } & 19.492 & 0.341 & .000 & 1.390 & 0.315 & .001 & 3.348 & 0.231 & .000 \\ \text { Age } & 0.019 & 0.009 & .039 & 0.008 & 0.006 & .205 & 0.002 & 0.004 & .600 \\ \text { Group } & 0.297 & 0.251 & .237 & 0.294 & 0.163 & .072 & 0.047 & 0.106 & .659 \\ \text { Time } & 0.357 & 0.153 & .020 & -0.094 & 0.095 & .324 & -0.166 & 0.060 & .006 \\ \text { Sex (male) } & 0.156 & 0.287 & .587 & -0.129 & 0.187 & .490 & -0.295 & 0.121 & .015 \\ \text { Group x Time } & \mathbf{0 . 3 1 3} & \mathbf{0 . 2 2 0} & .156 & \mathbf{0 . 3 6 0} & \mathbf{0 . 1 3 7} & .009 & \mathbf{0 . 0 2 4} & \mathbf{0 . 0 8 7} & .779 \\ \text { Group x Sex } & -0.242 & 0.405 & .552 & -0.219 & 0.264 & .407 & -0.140 & 0.171 & .414 \\ \text { Time x Sex } & 0.185 & 0.252 & .463 & 0.236 & 0.157 & .133 & -0.019 & 0.099 & .848 \\ \text { Group x Time x Sex } & 0.362 & 0.357 & .312 & 0.006 & 0.222 & .979 & -0.117 & 0.141 & .407\end{array}$

Predicted preference for each image in the manipulation phase was calculated based on ratings during the pre-test phase; a cubic regression was run on image BMI vs attractiveness rating in pre-test trials and predicted rating was then calculated for each manipulation phase trial. The difference between the predicted and actual rating was then used as the outcome variable and data were analysed using ImerTest in $\mathrm{R}$ as before. Sample, participant, original image identity, and specific image viewed were entered as cross-classified random effects. Trial type (manipulation trial or distractor) and trial position, and the interaction between the two were entered as fixed effects. Results for the fixed effects are shown in Table 4. For participants in the underweight bodies condition, there was a small significant interaction such that later manipulation trials were rated as significantly more attractive than earlier manipulation trials. For participants in the obese bodies condition, there was a significant impact of trial position on all images such that later images were rated as more attractive, however a 
borderline interaction with trial type suggested this impact was potentially reduced in the (obese) manipulation stimuli.

Table 4. Effects of trial position on rated attractiveness of manipulation stimuli in Study 3. (Trial type indicates manipulation stimuli 1, vs distractors 0 )

\begin{tabular}{lllllll} 
& & $\boldsymbol{B}$ & $\boldsymbol{S E}$ & $\boldsymbol{d f}$ & $\boldsymbol{t}$ & $\boldsymbol{p}$ \\
\hline Group 1: & (Intercept) & 0.017 & 0.315 & 3.2 & 0.054 & .960 \\
underweight & Trial position & 0.003 & 0.003 & 3082 & 0.899 & .369 \\
bodies condition & Trial type & 0.039 & 0.227 & 72.1 & 0.173 & .863 \\
& Position x trial type & 0.013 & 0.004 & 3088 & 3.296 & .001 \\
& & & & & & \\
Group 2: obese & (Intercept) & -0.038 & 0.145 & 67 & -0.261 & .795 \\
bodies condition & Trial position & 0.013 & 0.003 & 2273 & 4.002 & .000 \\
& Trial type & 0.217 & 0.164 & 54.8 & 1.318 & .193 \\
& Position x trial type & -0.007 & 0.004 & 2279 & -1.859 & .063
\end{tabular}

\section{Meta-Regression of Studies 1 and 3}

All data from Studies 1 and 3 were entered into a series of meta-analytic models using ImerTest in R. Study, sample and participant were entered as random effects, with age as a fixed effect covariate, and time, group and participant sex entered as interacting fixed effects. As shown in Table 3, these models yielded a significant interaction for time and group for preferences at BMI $30 \mathrm{~kg} / \mathrm{m}^{2}$, but not for peak BMI preference or preference at BMI $16 \mathrm{~kg} / \mathrm{m}^{2}$. It is worth noting that the regression coefficient value for the interaction term on BMI $30 \mathrm{~kg} / \mathrm{m}^{2}$ is similar across both studies (close to .3; except for the female participants in Study 1 Sample 2) and that the lack of a significant result in Study 3 was driven by the larger error for this term, i.e. the data were noisier, which may be reasonable given the lower number of manipulation stimuli and the presence of distractors.

\section{General Discussion}

The current studies aimed to test the manner in which visual exposure to exemplars of very low or very high weight bodies changes representations of body weight attractiveness. Cross-cultural variation in body ideals clearly shows patterns of differing non-linear and asymmetric attitudes to weight around that sample's 'ideal' (e.g., attractiveness ratings of bodies 'heavier than ideal' dropping sharply in the UK vs staying high in South Africa or rural Nicaragua: Boothroyd et al., 2016; Tovee et al., 2006). These patterns, however, may arise from local cultural associations with weight and it was not clear whether 'mere' visual exposure could adjust preferences in this non-linear fashion. Our studies therefore sought to test the effects of exposure to bodies at one weight extreme or the other on preferences for bodies across the natural range of female BMI.

Concordant results from Study 1 and Study 2, which used different methods and different adapting stimuli, combine to suggest that visual exposure impacts on body ideals may have primarily 'local' impacts. The results for the underweight-bodies manipulation trials in Study 3 likewise support this. Study 3 as a whole, however, showed less clear results: there was weak potential evidence of a shift in central ideal, but no evidence of the changes in predicted ratings at BMI 16 or $30 \mathrm{~kg} / \mathrm{m}^{2}$ that ought to accompany a function shift. Overall results from Studies 1 and 3 combined, however, continued to show support for an effect of adaptation on preferences for overweight/obese bodies, but not function peak or preferences for underweight bodies.

The difference in main results between Studies 1 and 3 could ostensibly arise from the fact that adapting stimuli in Study 1 were aspirational, while those in Study 3 were not, which would suggest that the impacts in Study 1 were in fact driven by associative learning. However, stimuli in Study 2 were also non-aspirational. Furthermore, Boothroyd et al (2012) found no difference in the magnitude of effects of adapting to aspirational or non-aspirational stimuli on subsequent weight 
preferences (see also Stephen \& Perera, 2014). It is important to note, however, that the earlier study used identical methods in the aspirational and non-aspirational studies. In Study 3 of the current paper, 'distractor' bodies were used in the adaptation phase in order to obscure the nature of the manipulation from participants who might otherwise have more obviously noticed a long string of obese bodies in what was meant to be a continuous display. Thus, although the adaptation phase was a similar length to Study 1 and Boothroyd et al (2012), there were fewer adapting stimuli overall. A future study may benefit from lengthening the adaptation phase and adding top-up adaptation trials within the post-test phase.

Crucially, across all three studies, there is minimal evidence for function shifting and ergo minimal evidence supporting the suggestion that conceptions of body weight attractiveness are tethered to a (single) prototype. The estimates of the interaction effect for peak BMI preference were mostly positive, but even a combined sample size of 373 participants across Studies 1 and 3 was still insufficient to yield a significant effect. In contrast, adaptation studies using single points from arrays or forced choice estimates of body size ideals are able to find significant impacts with fewer than 20 participants per condition (Boothroyd et al., 2012). We thus regard it as unlikely that a function shift could be responsible for those impacts. On the other hand, our data do support the suggestion that the changes in function shape seen between different cultural groups could arise through 'mere exposure' to differing numbers of high vs low weight bodies.

Other possible mechanisms for our results include, for instance, categorical impacts of stimuli. There is evidence that body weight is represented by at least two visual categories (British participants find it easier to distinguish bodies either side of approximately BMI $27 \mathrm{~kg} / \mathrm{m}^{2}$ than equivalent distances within the healthy/attractive or overweight/unattractive ranges: Tovee, Edmonds, \& Vuong, 2012) as well as separate encoding for fat and muscle (Sturman, Stephen, Mond, Stevenson, \& Brooks, 2017). It is possible, therefore, that viewing obese bodies activates the category 'overweight body' more generally, and that this activation leads to facilitated activation of that category subsequently, thus resulting in more positive valence via perceptual fluency (Reber, Winkielman, \& Schwarz, 1998). There is also evidence that differing categories of body (own vs other in this instance) may be represented hierarchically with some adaptation impacts being distinct between the two (Brooks, Mond, Stevenson, \& Stephen, 2016), and as such it may be possible to adapt bodies at one weight and not another.

Although some studies into the general perception of body weight have suggested a central prototype, other studies have found asymmetries in the malleability of this norm (Hummel et al., 2013; Winkler \& Rhodes, 2005). Furthermore, fMRI data have shown currently unexplained activation patterns for body weight adaptation outside of the typical perceptual areas where bodies are encoded - specifically in the left pre-central gyrus (Hummel et al., 2013). Such results are hard to explain given the association of the LPCG with the motor system, but this pattern was also observed in participants adapted to attractive faces (Fu et al., 2014), which suggests that adaptation on cues to attractiveness (including weight) may act on factors beyond the perceptual system, even where the adapting stimuli themselves are neutrally valenced.

Caution should be taken with these results insofar as the Chinese sample in Study 3 was comparatively underpowered (although still with sufficient participants to detect an interaction effect of a similar magnitude to that seen by Boothroyd et al., 2012) and participants in all studies are predominantly educated urban residents in just two countries. Furthermore, the current studies focused only on judgements of attractiveness and did not investigate the direct links between changes in preferences versus changes in perceived veridicality or normality. Further research should examine how changes in distribution of 'attractiveness' in adaptation-like paradigms links to changes in other aspects of perception. For instance, in facial attraction, DeBruine, Jones, Unger, Little, and Feinberg (2007) have demonstrated that 'normality' and 'attractiveness' may yield independent after effects. Considering other aspects of body perception in a manner which goes beyond point estimates/category boundaries may also highlight better the mechanisms at play (see e.g., Cornelissen, Gledhill, Cornelissen, \& Tovee, 2016). 
Previous research has tended to assume that changes in body size ideals after exposure to bodies at one weight extreme is due to a shift in the underlying 'prototype' or 'norm' from which body perceptions are derived. The current studies, however, give the first evidence that the more 'localised' differences in body preferences across the weight spectrum seen between individuals in different cultural contexts can be induced in experimental participants through visual exposure to body stimuli. Our finding of more 'local' effects of exposure on weight ideals has implications, for example, for discussions of how media images impact body ideals and body image in women and suggest that further research is required to understand how 'mere exposure' effects impact affective judgements of bodies.

References

Anzures, G., Mondloch, C. J., \& Lackner, C. (2009). Face Adaptation and Attractiveness Aftereffects in 8-Year-Olds and Adults. Child Development, 80(1), 178-191. doi:10.1111/j.1467-8624.2008.01253.x

Bestelmeyer, P. E. G., Jones, B. C., DeBruine, L. M., Little, A. C., Perrett, D. I., Schneider, A., . . Conway, C. A. (2008). Sexcontingent face aftereffects depend on perceptual category rather than structural encoding. Cognition, 107(1), 353365.

Boothroyd, L. G., Jucker, J. L., Thornborrow, T., Jamieson, M. A., Burt, D. M., Barton, R. A., . . Tovee, M. J. (2016). Television exposure predicts body size ideals in rural Nicaragua. British Journal of Psychology, 107(4), 752-767. doi:10.1111/bjop.12184

Boothroyd, L. G., Meins, E., Vukovic, J., \& Burt, D. M. (2014). Developmental changes in children's facial preferences. Evolution and Human Behavior, 35(5), 376-383. doi:10.1016/j.evolhumbehav.2014.05.002

Boothroyd, L. G., Tovee, M. J., \& Pollet, T. V. (2012). Visual Diet versus Associative Learning as Mechanisms of Change in Body Size Preferences. Plos One, 7(11), e48691. doi:10.1371/journal.pone.0048691

Brooks, K. R., Mond, J. M., Stevenson, R. J., \& Stephen, I. D. (2016). Body Image Distortion and Exposure to Extreme Body Types: Contingent Adaptation and Cross Adaptation for Self and Other. Frontiers in Neuroscience, 10, 334. doi:10.3389/fnins.2016.00334

Cornelissen, K. K., Gledhill, L. J., Cornelissen, P. L., \& Tovee, M. J. (2016). Visual biases in judging body weight. British Journal of Health Psychology, 21(3), 555-569. doi:10.1111/bjhp.12185

de Haan, M., Johnson, M. H., Maurer, D., \& Perrett, D. I. (2001). Recognition of individual faces and average face prototypes by 1-and 3-month-old infants. Cognitive Development, 16(2), 659-678.

DeBruine, L. M., Jones, B. C., Unger, L., Little, A. C., \& Feinberg, D. R. (2007). Dissociating averageness and attractiveness: Attractive faces are not always average. Journal of Experimental Psychology-Human Perception and Performance, 33(6), 1420-1430. doi:10.1037/0096-1523.33.6.1420

Fu, G. Y., Mondloch, C. J., Ding, X. P., Short, L. A., Sun, L. P., \& Lee, K. (2014). The neural correlates of the face attractiveness aftereffect: A functional near-infrared spectroscopy (fNIRS) study. Neuroimage, 85, 363-371. doi:10.1016/j.neuroimage.2013.04.092

Griffey, J. A. F., \& Little, A. C. (2014). Infant's visual preferences for facial traits associated with adult attractiveness judgements: Data from eye-tracking. Infant Behavior \& Development, 37(3), 268-275. doi:10.1016/j.infbeh.2014.03.001

Hummel, D., Rudolf, A. K., Brandi, M. L., Untch, K. H., Grabhorn, R., Hampel, H., \& Mohr, H. M. (2013). Neural Adaptation to Thin and Fat Bodies in the Fusiform Body Area and Middle Occipital Gyrus: An fMRI Adaptation Study. Human Brain Mapping, 34(12), 3233-3246. doi:10.1002/hbm.22135

Kuznetsova, A., Brockhoff, P. B., \& Christensen, R. H. B. (2016). Tests in Linear Mixed Effects Models. Retrieved from http://cran.uib.no/web/packages/ImerTest/ImerTest.pdf

Langlois, J. H., Roggman, L. A., Casey, R. J., Ritter, J. M., Rieserdanner, L. A., \& Jenkins, V. Y. (1987). Infant Preferences for Attractive Faces - Rudiments of a Stereotype. Developmental Psychology, 23(3), 363-369.

Mo, J. J. Y., Cheung, K. W. K., Gledhill, L. J., Pollet, T. V., Boothroyd, L. G., \& Tovee, M. J. (2014). Perceptions of Female Body Size and Shape in China, Hong Kong, and the United Kingdom. Cross-Cultural Research, 48(1), 78-103. doi:10.1177/1069397113510272

Nishimura, M., Maurer, D., Jeffery, L., Pellicano, E., \& Rhodes, G. (2008). Fitting the child's mind to the world: adaptive normbased coding of facial identity in 8-year-olds. Developmental Science, 11(4), 620-627. doi:10.1111/j.14677687.2008.00706.x

Palumbo, R., Laeng, B., \& Tommasi, L. (2013). Gender-specific aftereffects following adaptation to silhouettes of human bodies. Visual Cognition, 21(1), 1-12. doi:10.1080/13506285.2012.753970

Penton-Voak, I. S., Bate, H., Lewis, G., \& Munafo, M. R. (2012). Effects of emotion perception training on mood in undergraduate students: randomised controlled trial. British Journal of Psychiatry, 201(1), 71-72. doi:10.1192/bjp.bp.111.107086

R Development Core Team. R: A Language and Environment for Statistical Computing. Vienna, Austria: R Foundation for Statistical Computing.

Ramsey-Rennels, J. L., \& Langlois, J. H. (2006). Infants' differential processing of female and male faces. Current Directions in Psychological Science, 15(2), 59-62. 
Reber, R., Winkielman, P., \& Schwarz, N. (1998). Effects of perceptual fluency on affective judgments. Psychological Science, 9(1), 45-48. doi:10.1111/1467-9280.00008

Rhodes, G., Geddes, K., Jeffery, L., Dziurawiec, S., \& Clark, A. (2002). Are average and symmetric faces attractive to infants? Discrimination and looking preferences. Perception, 31(3), 315-321.

Rhodes, G., Jeffery, L., Clifford, C. W. G., \& Leopold, D. A. (2007). The timecourse of higher-level face aftereffects. Vision Research, 47(17), 2291-2296.

Slater, A., Quinn, P. C., Hayes, R., \& Brown, E. (2000). The role of facial orientation in newborn infants' preference for attractive faces. Developmental Science, 3(2), 181-185. doi:doi:10.1111/1467-7687.00111

Slater, A., Von der Schulenburg, C., Brown, E., Badenoch, M., Butterworth, G., Parsons, S., \& Samuels, C. (1998). Newborn infants prefer attractive faces. Infant Behavior \& Development, 21(2), 345-354.

Stephen, I. D., \& Perera, A. T. M. (2014). Judging the differences between women's attractiveness and health: Is there really a difference between judgments made by men and women? Body Image, 11(2), 183-186. doi:10.1016/j.bodyim.2013.11.007

Sturman, D., Stephen, I. D., Mond, J., Stevenson, R. J., \& Brooks, K. R. (2017). Independent Aftereffects of Fat and Muscle: Implications for neural encoding, body space representation, and body image disturbance. Scientific Reports, 7, 40392. doi:10.1038/srep40392

Tovee, M. J., \& Cornelissen, P. L. (2001). Female and male perceptions of female physical attractiveness in front-view and profile. British Journal of Psychology, 92, 391-402.

Tovee, M. J., Edmonds, L., \& Vuong, Q. C. (2012). Categorical perception of human female physical attractiveness and health. Evolution and Human Behavior, 33(2), 85-93. doi:10.1016/j.evolhumbehav.2011.05.008

Tovee, M. J., Maisey, D. S., Emery, J. L., \& Cornelissen, P. L. (1999). Visual cues to female physical attractiveness. Proceedings of the Royal Society of London Series B-Biological Sciences, 266(1415), 211-218.

Tovee, M. J., Swami, V., Furnham, A., \& Mangalparsad, R. (2006). Changing perceptions of attractiveness as observers are exposed to a different culture. Evolution and Human Behavior, 27(6), 443-456. doi:10.1016/j.evolhumbehav.2006.05.004

Winkler, C., \& Rhodes, G. (2005). Perceptual adaptation affects attractiveness of female bodies. British Journal of Psychology, 96, 141-154. doi:10.1348/000712605×36343 
Electronic supplementary material 1. Hypothetical data demonstrating function shift vs localised change.

\begin{tabular}{|c|c|c|c|}
\hline BMI & Original rating & Peak shift & Localised increase $>30$ \\
\hline 11 & 1 & 1 & 1 \\
\hline 12 & 1.7 & 1 & 1.7 \\
\hline 13 & 2.4 & 1 & 2.4 \\
\hline 14 & 3.1 & 1.7 & 3.1 \\
\hline 15 & 3.8 & 2.4 & 3.8 \\
\hline 16 & 4.5 & 3.1 & 4.5 \\
\hline 17 & 5.2 & 3.8 & 5.2 \\
\hline 18 & 5.9 & 4.5 & 5.9 \\
\hline 19 & 6.6 & 5.2 & 6.6 \\
\hline 20 & 7 & 5.9 & 7 \\
\hline 21 & 6.5 & 6.6 & 6.5 \\
\hline 22 & 6 & 7 & 6 \\
\hline 23 & 5.5 & 6.5 & 5.5 \\
\hline 24 & 5 & 6 & 5 \\
\hline 25 & 4.5 & 5.5 & 4.5 \\
\hline 26 & 4 & 5 & 4 \\
\hline 27 & 3.5 & 4.5 & 3.5 \\
\hline 28 & 3 & 4 & 3 \\
\hline 29 & 2.5 & 3.5 & 2.5 \\
\hline 30 & 2 & 3 & 3 \\
\hline 31 & 1.75 & 2.5 & 2.75 \\
\hline 32 & 1.75 & 2 & 2.75 \\
\hline 33 & 1.5 & 1.75 & 2.5 \\
\hline 34 & 1.5 & 1.75 & 2.5 \\
\hline 35 & 1.25 & 1.5 & 2.25 \\
\hline 36 & 1.25 & 1.5 & 2.25 \\
\hline 37 & 1 & 1.25 & 2 \\
\hline 38 & 1 & 1.25 & 2 \\
\hline 39 & 1 & 1 & 2 \\
\hline 40 & 1 & 1 & 2 \\
\hline
\end{tabular}

Electronic supplementary material 2. R-Markdown script and data files for all analyses and figures.

Files available at: https://osf.io/7c6wt/

Body_Function_Adaptation_paper_analysesFINAL.Rmd - Analysis script

all_MLMs.csv - Data for Studies 1 and 3

goodfunctiondata_UK_mlm.csv - Study 2 data

Functionpaper_study1_layerdfig_data.csv - Study 1 figure data

Function_Study3figures.csv - Study 3 figure data 